\title{
BMJ Open In out-of-hospital cardiac arrest, is the positioning of victims by bystanders adequate for CPR? A cohort study
}

\author{
Patrick Wagner, ${ }^{1}$ Sebastian Schloesser, ${ }^{2}$ Julia Braun, ${ }^{3}$ Hans-Richard Arntz, ${ }^{4}$ \\ Jan Breckwoldt (i) ${ }^{5}$
}

To cite: Wagner $P$, Schloesser $S$, Braun J, et al. In out-ofhospital cardiac arrest, is the positioning of victims by bystanders adequate for CPR? A cohort study. BMJ Open 2020;10:e037676. doi:10.1136/ bmjopen-2020-037676

- Prepublication history for this paper is available online. To view these files, please visit the journal online (http://dx.doi. org/10.1136/bmjopen-2020037676).

Received 12 February 2020 Revised 27 July 2020 Accepted 13 August 2020

Check for updates

(C) Author(s) (or their employer(s)) 2020. Re-use permitted under CC BY-NC. No commercial re-use. See rights and permissions. Published by BMJ.

${ }^{1}$ Anesthesiology, CharitéMedical University of Berlin, Berlin, Germany

${ }^{2}$ Anaesthesiology, Helios Klinikum Emil von Behring, Berlin-Zehlendorf, Berlin, Germany

${ }^{3}$ Epidemiology, Biostatistics and Prevention Institute (EBPI), University of Zurich, Zurich, Switzerland

${ }^{4}$ Benjamin Franklin Medical Center, Department of Cardiology, Charite Medical Faculty Berlin, Berlin, Germany ${ }^{5}$ University Hospital Zurich, Institute of Anesthesiology, University of Zurich Faculty of Medicine, Zurich, Switzerland

Correspondence to

Dr Jan Breckwoldt;

jan.breckwoldt@usz.ch

\section{ABSTRACT}

Objectives Outcome from out-of-hospital cardiac arrest (OHCA) highly depends on bystander cardiopulmonary resuscitation (CPR) with high-quality chest compressions (CCs). Precondition is a supine position of the victim on a firm surface. Until now, no study has systematically analysed whether bystanders of OHCA apply appropriate positions to victims and whether the position is associated with a particular outcome.

Design Prospective observational cohort study.

Setting Metropolitan emergency medical services (EMS) serving a population of 400 000; dispatcher-assisted CPR was implemented. We obtained information from the first EMS vehicle arriving on scene and matched this with data from semi-structured interviews with witnesses of the arrest.

Participants Bystanders of all OHCAs occurring during a 12-month period (July 2006-July 2007). From 201 eligible missions, 200 missions were fully reported by EMS. Data from 138 bystander interviews were included.

Primary and secondary outcome measures Proportion of positions suitable for effective CCs; related survival with favourable neurological outcome at 3 months.

Results Positioning of victims at EMS arrival was 'supine on firm surface' in 64 cases (32.0\%), 'recovery position (RP)' in 37 cases (18.5\%) and other positions unsuitable for CCs in 99 cases (49.5\%). Survival with favourable outcome at 3 months was $17.2 \%$ when 'supine position' had been applied, $13.5 \%$ with 'RP' and $6.1 \%$ with 'other positions unsuitable for CCs'; a statistically significant association could not be shown $(p=0.740$, Fisher's exact test). However, after 'effective CCs' favourable outcome at 3 months was $32.0 \%$ compared with $5.3 \%$ if no actions were taken. The OR was $5.87(\mathrm{p}=0.02)$.

Conclusion In $\mathrm{OHCA}$, two-thirds of all victims were found in positions not suitable for effective CCs. This was associated with inferior outcomes. A substantial proportion of the victims was placed in RP. More attention should be paid to the correct positioning of victims in OHCA. This applies to CPR training for laypersons and dispatcherassisted CPR.

\section{INTRODUCTION}

Survival and favourable outcome after outof-hospital cardiac arrest (OHCA) substantially increase if bystander cardiopulmonary resuscitation (CPR) is performed, with a

\section{Strengths and limitations of this study}

Up to present, this is the first systematic analysis of the positions bystanders applied to victims of outof-hospital cardiac arrest (OHCA).

- All OHCAs within the reporting period of one total year were analysed, full data were retrieved for 200 out of 201 cases.

- The study covered a population of 400000 inhabitants (over 1 year).

- The study reports how bystander cardiopulmonary resuscitation relates to clinically relevant outcomes (3 months survival with favourable neurological outcome).

- Data collection was undertaken in 2006-2007, as part of a broader observational study.

twofold to fourfold gain in survival. ${ }^{1-3}$ Such outcomes have also been shown to be associated with CPR quality, ${ }^{4}$ which is most importantly ensured by high-quality chest compressions (CCs). A precondition to effectively performing CCs is to position the victim supine on a firm surface. However, it is not known how often bystanders in reallife OHCA situations choose appropriate positions for effective CCs. A recent PubMed search with the MeSH terms 'position' (OR) 'victim position' (AND) 'resuscitation', 'supine position' (AND) 'resuscitation' as well as 'supine' (AND) 'resuscitation' did not yield relevant results.

There have been indications that the positioning of OHCA victims may introduce serious problems. Recently, a group from Spain reported a case series of seven patients who had been placed into the recovery position (RP), although they were apparently in cardiac arrest when the emergency medical services (EMS) arrived. ${ }^{5}$ The group conducted a follow-up simulation study with medical students showing that respiratory arrest was detected significantly less frequently if the simulated patient was lying in RP rather than 
supine with head tilt and chin lift. ${ }^{6}$ Thus, the positioning of victims in RP might result in less favourable patient outcomes.

Concerns have also been raised with respect to CPR training, which may put too much weight on RP and might thereby mislead bystanders in real-life OHCA. ${ }^{7} \mathrm{RP}$ is part of many first aid/BLS training programmes ${ }^{89}$ and may therefore seem attractive as an option to lay bystanders during OHCA. This point is supported by a manikin study in which $21 \%$ of the participants placed the simulated OHCA victim into the RP. ${ }^{10}$ Furthermore, two large, representative interview studies have shown that laymen found placing the victim in the $\mathrm{RP}$ was an advisable reaction to a cardiac arrest. ${ }^{11}{ }^{12}$ In the first of these studies, conducted in the area of Greater London, $45 \%(452 / 1011)$ of the interviewees would have first tried to place an unconscious person in the RP. ${ }^{12}$ In the other study, a nationwide survey conducted in Norway (with a well-trained population) in 2014, the participants were asked how they would react in the case of an unconscious person not breathing normally ('but emitting short gasps'); 42\% (424/1000) suggested starting CPR while 33\% (326/1000) decided on RP. ${ }^{11}$

In response to the findings by Freire-Tellado $e t$ al, more data are needed to consolidate the knowledge on this topic. ${ }^{13}$ We therefore felt it was important to report additional data from a study our group published in 2009, at that time dealing with the perceptions of bystanders when witnessing OHCA. ${ }^{14}$ In that 2009 paper, we collected data on the positions in which victims had been found by the first EMS vehicle arriving on scene. In addition, the actions taken by bystanders were recorded. We combined these data with statements gathered from semi-structured interviews with the witnesses of the cardiac arrests. The current paper systematically reports the positions in which victims of OHCA were found on EMS arrival in that 2009 study. For secondary outcomes, the positioning of victims was related to patient outcomes, including 'return of spontaneous circulation' (ROSC), hospital discharge and Glasgow-Pittsburgh Cerebral Performance Categories (CPC) at 90 days. The current paper aims to provide more information on how bystanders act in OHCA and thus could help to better tailor CPR courses and EMS dispatch to the needs of lay rescuers.

\section{METHODS}

Over a 12-month period (5 July 2006-4 July 2007), we prospectively analysed all missions for CA of two physicianstaffed EMS units at Berlin, Germany, serving a population of approximately 400000 inhabitants. The EMS is organised as a two-tiered system with physician-staffed ALS units and paramedic-staffed BLS units. Dispatcherassisted CPR had been introduced in 2005, including instructions for positioning. However, at the time of the study, dispatcher-assisted CPR was offered only in around $20 \%-25 \%$ of cardiac arrests. ${ }^{14}$ Further details about the EMS have been published previously. ${ }^{14}$ We included all cases of cardiac arrest in which a bystander encountered a victim in need of resuscitation attempts (as assessed by the EMS physician on scene). We excluded arrests in the presence of EMS, those of traumatic origin, sudden infant death as well as situations where CPR was withheld based on the EMS physician's decision (eg, due to a terminal patient status).

The EMS physician of each mission collected structured information from the first EMS vehicle on scene. This information included the nature and the quality of the actions bystanders took, such as removal of clothes, positioning of the patient and CCs. CCs were classified by visual assessment as 'effective CPR', 'ineffective CPR', 'CPR reported (but not observed)' and 'no actions'. We defined 'effective CCs' as those where both, compression depth (neither 'too shallow' nor 'too deep') and frequency (neither 'too slowly' nor 'too quick') were judged being adequate. Missing data were completed after the mission by telephone consultation with the responsible paramedic of the first vehicle on scene. In addition, we conducted semi-structured interviews with the witnesses of the collapse, if they could be identified and were willing to be interviewed. The interviews were conducted face-to-face or by telephone, up to 3 days after the event. We obtained basic demographics, a description of the circumstances of the collapse, the actions taken by bystanders as well as the educational status and first aid training history (for further details see interview guide, online supplemental table 1) and our previous report. ${ }^{14}$ Patient characteristics were derived from EMS run charts, patients' outcomes from hospital reports and CPC after 3 months by telephone survey.

All original data are available on reasonable request. No external funding was given for this study.

\section{Patient and public involvement}

It was not appropriate to involve patients or the public in the design, conduct, reporting or dissemination plans of our research.

\section{Statistical analysis}

Answer categories were expressed as percentages, and continuous parameters as median, minimum, maximum and quartiles. Comparison of discrete parameters was performed by using Fisher's exact test, while a KruskalWallis test was conducted to analyse continuous variables (such as age) in relation to positioning of the victim, and CC quality. To quantify the relationship between CC quality or positioning and a favourable outcome, we used a logistic regression model. Note that the two influential variables are closely related, for which reason they could not be included in one joint model due to multicollinearity. We compared the two univariate models (positioning and CC quality) using Akaike's information criterion (AIC). An a priori power analysis was not performed because of the observational nature of the study. We used SPSS V.25.0, the statistics Python library (https://docs.scipy.org/doc/scipy/reference/stats. 
html) and R V.3.6.2 (https://cran.r-project.org) for the analyses. $\mathrm{P}$ values $<0.05$ were assumed to be statistically significant.

\section{RESULTS}

\section{Characteristics of patients and bystanders}

From 201 missions meeting the inclusion criteria, 200 reports by the first EMS vehicle on scene could be analysed. Of these cases, 135 (67.5\%) were witnessed, 43 (21.5\%) were unwitnessed and in the remaining cases it could not be clarified whether the arrest had been observed. The median age of male patients was 68 (25th-75th percentiles: 58-76) and 80.5 (68-85) for females. The incidence of initial ventricular fibrillation/ventricular tachycardia was $40.3 \%$; $73.9 \%$ of 199 arrests occurred at home. Bystanders were female in $60.1 \%$ of the cases, and female bystanders had a median age of 56, male bystanders of 47. Personal relation to the victim was generally close, and only $11.1 \%$ did not know the victim before the incident. In 31 of 138 interviews $(22.5 \%)$, the interviewees reported that dispatchers had offered instructions.

Pre-existing BLS knowledge was obtained from 134 interviewees: $14.9 \%$ had never attended a first aid course, $44.8 \%$ had completed at least one course and $22.4 \%$ were healthcare professionals, including $6.0 \%$ off-duty physicians. $^{14}$

\section{Positioning of the victim}

The positions of victims when the first EMS vehicle arrived are shown in table 1 . Positions were suitable for effective CCs in only one-third of the cases. The group of lay rescuer interviewees placed the victims supine on a

Table 1 Actions taken by bystanders observed by the first EMS vehicle on scene $(n=200)$

\begin{tabular}{lrl}
\hline Action & $\mathbf{n}$ & $\%$ \\
\hline Positioning & & \\
Supine on firm surface & 64 & $32.0 \%$ \\
\hline Position not suitable for CPR & 136 & $68.0 \%$ \\
$\quad$ RP & 37 & $18.5 \%$ \\
$\quad$ Other than RP & 99 & $49.5 \%$ \\
\hline Total & 200 & \\
CC quality & & \\
CCs effective* & 25 & $12.5 \%$ \\
\hline CCs not effective* & 21 & $10.5 \%$ \\
CCs reported but not observed $\dagger$ & 28 & $14.0 \%$ \\
\hline No actions taken & 95 & $47.5 \%$ \\
Missing information & 31 & $15.5 \%$ \\
\hline Total & 200 & \\
\hline
\end{tabular}

*As assessed by the first EMS vehicle on scene.

†According to interviews with bystanders.

$\mathrm{CC}$, chest compression; CPR, cardiopulmonary resuscitation;

EMS, emergency medical services; RP, recovery position. firm surface in only 30 of 104 (28.8\%) cases. Various positions unsuitable for CCs were observed in the remaining cases, such as lying in bed, face down on the floor, in RP or sitting in a chair, a driver's seat or a wheelchair. RP at EMS arrival was found in $18.5 \%$ of all cases. The interviews with witnesses revealed 20 additional cases (10.0\%) in which victims had temporarily been positioned into the RP but were found in other positions on EMS arrival.

\section{Quality of chest compressions}

CC performance was assessed in 169 cases. In 74 arrests (43.8\%), CCs were either directly observed by the EMS or plausibly reported by witnesses. EMS assessed 25 cases (14.8\%) as 'effective CCs'. After 'effective CCs' favourable neurological outcome was markedly higher $(32.0 \%$ of the patients) than if CCs were 'ineffective' (14.3\%), CCs were 'reported but not observed' (17.2\%), or no action was taken $(5.3 \%)$. Table 2 provides further details.

Fisher's exact test showed moderate evidence for a relation between CC quality and favourable neurological outcomes with a $\mathrm{p}$ value of 0.0238 . In the logistic regression model quantifying the influence of CC quality on a favourable outcome, we found moderate evidence for a positive effect of both, 'observed, but ineffective CCs' and 'observed effective CCs' ('observed, but ineffective': OR 8.8, $95 \%$ CI 1.35 to $78.39, \mathrm{p}=0.03$; 'observed and effective': OR 5.87, $95 \%$ CI 1.45 to $26.7, \mathrm{p}=0.02$ ). This means that the odds for a favourable outcome were almost 9 and almost 6 times higher if CCs were observed. No evidence for such an effect was found for unobserved CCs. Note that the CIs are quite wide due to the comparably small sample size.

\section{Patient outcome in relation to positioning of the victim}

With respect to favourable neurological outcome at 3 months, victims in 'supine position' showed the best outcomes (see table 2) when compared with all victims in positions unsuitable for CCs $(17.2 \%$ vs $8.1 \%)$ or to cases with 'positions unsuitable for CCs, other than in RP' $(6.1 \%)$. The differences in favourable neurological outcomes of victims who had been placed in RP were not as strikingly different (13.5\%). Fisher's exact test for a relation between positioning and favourable neurological outcomes was not significant $(\mathrm{p}=0.740)$, and also the logistic regression model for favourable outcome did not show evidence for an influence of the position on the outcome.

\section{Comparison between logistic regression models for favourable outcome}

Fisher's exact test showed a strong association between CC quality and positioning ( $<<0.0001$; as shown in online supplemental table 2), for which reason it was not possible to include both variables in a joint model. A comparison of the model with positioning and the model with $\mathrm{CC}$ quality using AIC clearly preferred the model with CC quality (AIC 75.8 for CC quality vs 84.6 for positioning). 
Table 2 Actions of bystanders in relation to patient outcomes

Outcomes

\begin{tabular}{|c|c|c|c|c|c|c|c|}
\hline \multirow[b]{3}{*}{ Action taken } & & \multicolumn{6}{|l|}{ Outcomes } \\
\hline & & \multirow[b]{2}{*}{$\begin{array}{l}\text { Initial rhythm } \\
\text { VF/VT* }\end{array}$} & \multirow[b]{2}{*}{ ROSC } & \multirow[b]{2}{*}{$\begin{array}{l}\text { Admission } \\
\text { to hospital† }\end{array}$} & \multirow[b]{2}{*}{$\begin{array}{l}\text { Hospital } \\
\text { discharge }\end{array}$} & \multicolumn{2}{|c|}{ CPC at 3 months } \\
\hline & & & & & & $\begin{array}{l}\text { CPC } \\
1-2\end{array}$ & $\begin{array}{l}\text { CPC } \\
3-5\end{array}$ \\
\hline \multicolumn{8}{|l|}{ Positioning } \\
\hline Supine on firm surface & 64 & $51.5(33)$ & $40.6(26)$ & $39.1(25)$ & $18.8(12)$ & $17.2(11)$ & $21.9(14)$ \\
\hline $\mathrm{RP}$ & 37 & $45.9(17)$ & $48.6(18)$ & $45.9(17)$ & $21.6(8)$ & $13.5(5)$ & $35.1(13)$ \\
\hline \multirow[t]{2}{*}{ Other than RP } & 99 & $30.3(30)$ & $18.2(18)$ & $15.2(15)$ & $6.1(6)$ & $6.1(6)$ & $10.1(10)$ \\
\hline & 200 & & & & & & \\
\hline \multicolumn{8}{|l|}{ CC quality } \\
\hline CCs observed, effectiveł & 25 & $56.0(14)$ & $56.0(14)$ & $52.0(13)$ & $36.0(9)$ & $32.0(8)$ & $4.0(6)$ \\
\hline
\end{tabular}

Fisher's exact test for an association between position and CPC: $p=0.740$.

Fisher's exact test for an association between CC quality and CPC: $p=0.0238$.

${ }^{*}$ As assessed by the first EMS vehicle on scene (data from AEDs).

†CPC unknown: $\mathrm{n}=3$.

$\ddagger$ As assessed by the first EMS vehicle on scene.

§According to interviews with bystanders.

AED, Automated External Defibrillator; CC, chest compression; CPC, Glasgow-Pittsburgh Cerebral Performance Categories; CPR, cardiopulmonary resuscitation; EMS, emergency medical services; ROSC, return of spontaneous circulation; RP, recovery position; VF, ventricular fibrillation; VT, ventricular tachycardia.

We also found descriptive differences between the patient groups in terms of the proportion of shockable initial rhythms (see also table 2). Shockable rhythms were most likely if the victim was in a supine position and if bystanders had started CCs, whereas they were least likely if bystanders had not taken any action. However, no statistically significant differences could be demonstrated.

\section{Influence of bystander's gender and age}

As shown in table 3 , male bystanders were more likely to start CCs than females, although statistical significance was not reached $(\mathrm{n}=138$ cases; male bystanders started in $49.0 \%$, females in $38.5 \%$; $\mathrm{p}=0.221$ ). A significant association was found for the relation between gender and position $(p=0.016)$, where positions not suitable for CCs occurred more often with female bystanders. For CC quality we found no evidence for an association $(\mathrm{p}=0.332)$.

With respect to the bystander's age, a couple of descriptive differences were found in favour of younger rescuers (see table 3). Younger bystanders applied supine positions more often, removed clothes from the chest more often and applied 'positions not suitable for CCs, other than RP' less frequently than older rescuers. Younger bystanders also started CCs more often than older bystanders (see table 3). 'Effective CCs' were only observed for bystanders younger than 60 years of age. Bystanders who did not take any action at all were older than active bystanders, regardless of the bystander's gender. Overall, Kruskal-Wallis tests showed a strong association of age with positioning $(\mathrm{p}<0.001)$ and CC quality $(\mathrm{p}<0.001)$.

\section{Influence of medical knowledge}

For 104 lay bystanders and 30 healthcare workers, CPR training status was able to be analysed along with the positioning of the victim and to the quality of CCs (see table 4). For these persons, the positioning of the victim was highly dependent on BLS knowledge. Healthcare workers were more likely to apply positions suitable for CCs than lay rescuers who had received training $<5$ years before, $>5$ years before or had never attended a training (healthcare workers vs all lay rescuers: $63.3 \%$ vs $28.8 \%$ ). Furthermore, CPR was started more often by lay bystanders who had received training in the past 5 years than by bystanders who had taken courses $>5$ years before or by those without CPR training.

Finally, positioning into RP differed according to the BLS training status (see table 4): lay bystanders who had attended a course within the past 5 years applied the RP most frequently $(34.8 \%)$ than lay rescuers with courses from $>5$ years before or those without any training.

\section{DISCUSSION}

In this study, bystanders placed only one-third of the patients with cardiac arrest in positions suitable for effective CCs. To our knowledge, this is the first systematic report 
Table 3 Actions of bystanders in relation to gender and age (from 138 interviews)

\begin{tabular}{|c|c|c|c|c|c|}
\hline \multirow{3}{*}{ Action taken } & \multicolumn{2}{|c|}{ Gender $(n=138)$} & \multicolumn{3}{|c|}{ Age* $^{\star}(n=136)$} \\
\hline & Male & Female & $<30$ years & $30-60$ years & $>60$ years \\
\hline & $\%(n)$ & $\%(n)$ & $\%(n)$ & $\%(n)$ & $\%(n)$ \\
\hline \multicolumn{6}{|l|}{ Positioning } \\
\hline Position supine, firm surface & $47.3 \%(26)$ & $27.7 \%(23)$ & $69.2 \%(9)$ & $48.7 \%(37)$ & $6.4 \%(3)$ \\
\hline Position not suitable for CCs: RP & $25.5 \%(14)$ & $18.1 \%(15)$ & $23.1 \%(3)$ & $19.7 \%(15)$ & $21.3 \%(10)$ \\
\hline Position not suitable for CCs, other than RP & $27.3 \%(15)$ & $54.2 \%(45)$ & $7.7 \%(1)$ & $31.6 \%(24)$ & $72.3 \%(34)$ \\
\hline \multicolumn{6}{|l|}{ CC quality } \\
\hline CCs observed, effective† & $20.0 \%(11)$ & $12.0 \%(10)$ & $23.1 \%(3)$ & $23.7 \%(18)$ & $0.0 \%(0)$ \\
\hline CCs observed, not effective† & $14.5 \%(8)$ & $8.4 \%(7)$ & $15.4 \%(2)$ & $15.8 \%(12)$ & $2.1 \%(1)$ \\
\hline
\end{tabular}

Association between 'gender' and 'position': $p=0.016$ (Fisher's exact test).

Association between 'gender' and 'CC quality': $p=0.332$ (Fisher's exact test).

Association between 'age' and 'position': $p<0.001$ (Kruskal-Wallis test).

Association between 'age' and 'CC quality': $p<0.001$ (Kruskal-Wallis test).

${ }^{*}$ Data for age missing for $\mathrm{n}=2$.

†As assessed by the first EMS vehicle on scene.

$\ddagger$ Sum of 'CCs observed, effective', 'CCs observed, not effective', 'CCs not observed, but reported'.

$\mathrm{CC}$, chest compression; RP, recovery position.

on the positioning of cardiac arrest victims by bystanders under real-life conditions. It has to be acknowledged that the findings date back more than a decade, and the figure of one-third may well be higher today due to advanced dispatching technologies and experience. However, the Berlin EMS had already implemented dispatcher-assisted CPR at the time of the study, and had (during the study period) provided advice for positioning in $20 \%-25 \%$ of

Table 4 Actions of bystanders in relation to CPR training status, available from $n=134$ interviewees

Lay persons

Time since most recent CPR training

\begin{tabular}{|c|c|c|c|c|}
\hline & $\begin{array}{l}\text { No training ever } \\
\mathrm{n}=27\end{array}$ & $\begin{array}{l}>5 \text { years } \\
n=54\end{array}$ & $\begin{array}{l}\text { Up to } \\
5 \text { years } \\
n=23\end{array}$ & $\begin{array}{l}\text { Healthcare workers } \\
n=30\end{array}$ \\
\hline Action taken & $\%(n)$ & $\%$ (n) & $\%(n)$ & $\%(n)$ \\
\hline \multicolumn{5}{|l|}{ Positioning } \\
\hline Clothes removed from chest & $7.4 \%(2)$ & $18.5 \%(10)$ & $8.7 \%(2)$ & $50.0 \%(15)$ \\
\hline Position supine, firm surface & $14.8 \%(4)$ & $27.8 \%(15)$ & $47.8 \%(11)$ & $63.3 \%(19)$ \\
\hline $\mathrm{RP}$ & $18.5 \%(5)$ & $22.2 \%(12)$ & $34.8 \%(8)$ & $10.0 \%(3)$ \\
\hline Other than RP & $66.7 \%(18)$ & $27.8 \%(15)$ & $17.4 \%(4)$ & $26.7 \%(8)$ \\
\hline \multicolumn{5}{|l|}{ CC quality } \\
\hline CCs observed, effective* & $3.7 \%(1)$ & $5.6 \%(3)$ & $26.1 \%(6)$ & $36.7 \%(11)$ \\
\hline
\end{tabular}

${ }^{*}$ As assessed by the first EMS vehicle on scene.

$\mathrm{CC}$, chest compression; CPR, cardiopulmonary resuscitation; RP, recovery position. 
cases. ${ }^{14}$ Moreover, the bystander-CPR rate of $37 \%$ in our data set was not much lower than recent data from all over Europe which shows a range of $18 \%-82 \%$ (46.4\% for Germany). ${ }^{15}$ Our findings are supported by observations from everyday EMS experience and from a case series from Spain. ${ }^{5}$ Although potential influences are multifactorial and should be interpreted with caution, the figures should raise our awareness. Patients in supine positions were able to receive effective CCs, which was associated with improved neurological outcomes at 3 months. Even if conditions today might have improved in some settings, our findings may be seen as a snapshot with which further developments could be compared.

One important reason for the high incidence of unsatisfactory positions is the difficulty to confirm cardiac arrest, especially for laymen. ${ }^{10}$ This situation is aggravated by the confusing clinical signs of agonal breathing, ${ }^{14-16}$ which are common in OHCA. ${ }^{19}$ These circumstances may partly explain why victims were actively placed into RP. As a further point, bystanders might perceive the RP as a stable end point of their reactions to the collapse, not realising that they arrived at a dead-end; $18.5 \%$ of victims remained in RP until EMS arrival and for these cases unconsciousness and the absence of breathing were reported by the EMS. While a number of patients may have initially been at consciousness, deterioration of their condition had not been noticed. This point is supported by the experimental finding that respiratory arrest was less likely detected if the victim was in $\mathrm{RP}^{20}$

In addition to those victims who remained in RP until EMS arrival, a further $10.0 \%$ were positioned into RP intermittently and were then placed in supine position before EMS arrival. For some of these patients, it was plausibly reported that they still had shown signs of life after collapse, indicating the potential initial presence of circulation (and hence better starting conditions for favourable outcomes). The finding that patients in RP did not show substantially worse outcomes than those in supine positions supports this interpretation. Furthermore, RP patients showed a higher proportion of shockable rhythms than the group of patients in 'unsuitable positions other than RP'. In contrast, in patients with a clear absence of breathing (and inferior starting conditions), it may have been easier for bystanders to confirm CA and initiate CPR. Nonetheless, according to the interview comments, it is still likely that in a substantial proportion of the collapses, agonal breathing was misinterpreted as normal breathing and the initial decision for RP was not correct. In summary, it can be concluded that making the appropriate decision for or against RP appears to be difficult for lay rescuers. Accordingly, more attention should be paid to this issue in CPR training. ${ }^{78}$ In real-life situations, EMS dispatch should be able to build on CPR training content to assure proper positioning for CPR. ${ }^{121}$ To advance the knowledge in this field, it is clearly necessary to collect more systematic data on the positioning of victims.
Another relevant point arising from our data is that supine positions were applied more frequently, and CPR was started more likely if bystanders had more recently completed first aid training. This offers strong support for the effectiveness of CPR training for laymen, which is clearly linked to improved patient outcome. However, some concern may arise from the finding that the use of RP was also more frequent if laymen had completed their training more recently. This could potentially be explained by the idea that too much time had been spent on the RP during training, ${ }^{7}$ or that the RP had been taught in more meaningful contexts ${ }^{8}$ with the consequence of bystanders being more confident in applying RP than in performing CCs. ${ }^{12}$ RP could also be perceived as less harmful to a victim, fuelled by the lay rescuers' frequent fear of injuring the victim. ${ }^{22}{ }^{23}$ Finally, knowledge of the RP might distract bystanders from sufficient resuscitation, an interpretation that may be supported by cognitive load theory. ${ }^{24}$

All of these considerations indicate that more attention should be paid to the potentially adverse effects of CPR training for laymen. As their main learning objectives, courses should stress that doing nothing is much more harmful than applying (unnecessary) CCs. If courses aim to strengthen the focus on high-quality CCs, they should emphasise that the appropriate positioning of the victim is a vital precondition. This point should also be emphasised by EMS dispatchers in real-life cardiac arrests who should provide clear instructions for the proper positioning of victims.

The findings in respect to gender and age are in line with the literature. ${ }^{2526}$ For elderly rescuers, positioning victims and starting CCs could be difficult tasks to perform, due to physical incapacitation, a lack of knowledge or slower reaction times. Therefore, our results also call for CPR courses that are specifically tailored to elderly (female) rescuers.

\section{Limitations}

This is a single-centre study from a German metropolitan area. However, the reports from Spain and the abovementioned findings from Great Britain and Norway suggest similar conditions in other countries. Nevertheless, results can only be generalised with caution. As a second limitation, the data from interviews are subject to a significant selection bias on the part of the interviewed cohort: in our first publication on this topic, we found indicators for worse outcomes in the population of bystanders who were not available for an interview. ${ }^{14}$ It is very likely that this is also the case in this study. Third, CC quality was not objectively measured, and EMS personnel might have overestimated bystander performance. However, we did not want to delay high-quality CPR by EMS, and our aim was only to compare the magnitudes of CPR performance to each other. Fourth, the sample size was rather low for the cases in which associations were established between positioning and neurological outcome at 3 months. As a final limitation, we have to acknowledge that the data 
of this study were collected some years ago. However, we provide the first systematic analysis about how victim position may affect outcomes, and in the area of lay person training the content has not changed substantially over time. $^{78}$

\section{CONCLUSION}

In OHCA in a German metropolitan area only one-third of the patients was placed in a position suitable for effective CCs. Patients placed supine and receiving effective CCs showed better outcomes at 3 months. A significant proportion of the patients found in positions unsuitable for CPR remained in RP until EMS arrival, and this was more likely if bystanders had completed CPR training more recently. Knowledge of the RP might distract bystanders from performing sufficient resuscitation. We recommend paying far more attention to the positioning of victims during lay CPR courses. In addition, additional studies are needed to more thoroughly investigate the positioning of OHCA victims by bystanders.

Contributors PW is a resident at the Department of Anaesthesiology, Medical University of Berlin, Campus Benjamin Franklin, Berlin, Germany. He adapted and re-analysed the data sheet, conducted statistics and contributed to the manuscript. SS is a senior physician in the Department of Anaesthesiology, Helios Klinikum Emil von Behring, Berlin-Zehlendorf, Germany. He collected the data and generated the original data set. $\mathrm{JB}$ is a $\mathrm{PhD}$ and statistician scientist at the 'Epidemiology, Biostatistics and Prevention Institute' (EBPI) of the University of Zurich. She conducted the statistical work-up of the data set. HRA is former professor in cardiology in the Department of Internal Medicine II, Medical University of Berlin, Campus Benjamin Franklin, Berlin, Germany. He contributed to the study design and gave important intellectual input to the manuscript. JB was consultant in anaesthesiology at the Department of Anaesthesiology, Medical University of Berlin, Campus Benjamin Franklin, and is now employed at the Institute of Anaesthesiology, University of Zurich and Zurich University Hospital, Zurich, Switzerland. He designed the study and prepared the manuscript. Each author takes public responsibility for the entire work.

Funding The authors have not declared a specific grant for this research from any funding agency in the public, commercial or not-for-profit sectors.

Competing interests None declared.

Patient and public involvement Patients and/or the public were not involved in the design, or conduct, or reporting, or dissemination plans of this research.

Patient consent for publication Not required.

Ethics approval Approval for this study was given by the ethics committee of Charité-University Medicine Berlin (No. EA4/065/08).

Provenance and peer review Not commissioned; externally peer reviewed.

Data availability statement Data are available on reasonable request. Data are available on reasonable request from the corresponding author.

Open access This is an open access article distributed in accordance with the Creative Commons Attribution Non Commercial (CC BY-NC 4.0) license, which permits others to distribute, remix, adapt, build upon this work non-commercially, and license their derivative works on different terms, provided the original work is properly cited, appropriate credit is given, any changes made indicated, and the use is non-commercial. See: http://creativecommons.org/licenses/by-nc/4.0/.

\section{ORCID iD}

Jan Breckwoldt http://orcid.org/0000-0003-1716-1970

\section{REFERENCES}

1 Perkins GD, Travers AH, Berg RA, et al. Part 3: adult basic life support and automated external defibrillation: 2015 international consensus on cardiopulmonary resuscitation and emergency cardiovascular care science with treatment recommendations. Resuscitation 2015;95:e43-69.

2 Sasson C, Rogers MAM, Dahl J, et al. Predictors of survival from outof-hospital cardiac arrest: a systematic review and meta-analysis. Circ Cardiovasc Qual Outcomes 2010;3:63-81.

3 Wissenberg M, Lippert FK, Folke F, et al. Association of national initiatives to improve cardiac arrest management with rates of bystander intervention and patient survival after out-of-hospital cardiac arrest. JAMA 2013;310:1377-84.

4 Wik L, Steen PA, Bircher NG. Quality of bystander cardiopulmonary resuscitation influences outcome after prehospital cardiac arrest. Resuscitation 1994;28:195-203.

5 Freire-Tellado M, Pavón-Prieto MDP, Fernández-López M, et al. Does the recovery position threaten cardiac arrest victim's safety assessment? Resuscitation 2016;105:e1.

6 Navarro-Patón R, Freire-Tellado M, Fernández-González N, et al. What is the best position to place and re-evaluate an unconscious but normally breathing victim? a randomised controlled human simulation trial on children. Resuscitation 2019;134:104-9.

7 Wagner P, Lingemann C, Arntz H-R, et al. Official lay basic life support courses in Germany: is delivered content up to date with the guidelines? an observational study. Emerg Med J 2015;32:547-52.

8 Breckwoldt J, Lingemann C, Wagner P. Resuscitation training for lay persons in first responder courses. Transfer of knowledge, skills and attitudes. [German] (English translation: see Research Gate). Anaesthesist 2016;65:22-9.

9 Parnell MM, Larsen PD. Poor quality teaching in lay person CPR courses. Resuscitation 2007;73:271-8.

10 Piepho T, Resch N, Heid F, et al. Lay basic life support: the current situation in a medium-sized German town. Emerg Med J 2011;28:786-9.

11 Bakke HK, Steinvik T, Angell J, et al. A nationwide survey of first aid training and encounters in Norway. BMC Emerg Med 2017;17:6.

12 Donohoe RT, Haefeli K, Moore F. Public perceptions and experiences of myocardial infarction, cardiac arrest and CPR in London. Resuscitation 2006;71:70-9.

13 Handley AJ. Should we still be teaching the recovery position? Resuscitation 2017;115:A6-7.

14 Breckwoldt J, Schloesser S, Arntz H-R. Perceptions of collapse and assessment of cardiac arrest by bystanders of out-of-hospital cardiac arrest (OOHCA). Resuscitation 2009;80:1108-13.

15 Gräsner J-T, Wnent J, Herlitz J, et al. Survival after out-of-hospital cardiac arrest in Europe - Results of the EuReCa TWO study. Resuscitation 2020;148:218-26.

16 Bång A, Herlitz J, Martinell S. Interaction between emergency medical dispatcher and caller in suspected out-of-hospital cardiac arrest calls with focus on agonal breathing. A review of 100 tape recordings of true cardiac arrest cases. Resuscitation 2003;56:25-34.

17 Eisenberg MS. Incidence and significance of gasping or agonal respirations in cardiac arrest patients. Curr Opin Crit Care 2006;12:204-6.

18 Brinkrolf P, Metelmann B, Scharte C, et al. Bystander-witnessed cardiac arrest is associated with reported agonal breathing and leads to less frequent bystander CPR. Resuscitation 2018;127:114-8.

19 Rea TD. Agonal respirations during cardiac arrest. Curr Opin Crit Care 2005;11:188-91.

20 Freire-Tellado M, Navarro-Patón R, Pavón-Prieto MdelP, et al. Does lying in the recovery position increase the likelihood of not delivering cardiopulmonary resuscitation? Resuscitation 2017;115:173-7.

21 Lerner EB, Rea TD, Bobrow BJ, et al. Emergency medical service dispatch cardiopulmonary resuscitation prearrival Instructions to improve survival from out-of-hospital cardiac arrest: a scientific statement from the American heart association. Circulation 2012;125:648-55.

22 Savastano S, Vanni V. Cardiopulmonary resuscitation in real life: the most frequent fears of lay rescuers. Resuscitation 2011;82:568-71.

23 Coons SJ, Guy MC. Performing bystander CPR for sudden cardiac arrest: behavioral intentions among the general adult population in Arizona. Resuscitation 2009;80:334-40.

24 Chandler P, Sweller J. Cognitive load theory and the format of instruction. Cogn Instr 1991;8:293-332.

25 Vaillancourt C, Stiell IG, Wells GA. Understanding and improving low bystander CPR rates: a systematic review of the literature. CJEM 2008;10:51-65.

26 Dorph E, Wik L, Steen PA. Dispatcher-assisted cardiopulmonary resuscitation. An evaluation of efficacy amongst elderly. Resuscitation 2003;56:265-73. 